\title{
Application of D-Dimer Assay in Orthopaedic Trauma
}

\author{
Yongli Peng ${ }^{1 *}$, $\mathrm{Na} \mathrm{He}^{2}$ and Haojie Cui ${ }^{1}$ \\ ${ }^{1}$ The Third Department of Orthopedics, People's Hospital of Renqiu, China \\ ${ }^{2}$ Department of Clinical Laboratory, People's Hospital of Renqiu, China
}

Submission: March 07, 2017; Published: March 21, 2017

*Corresponding author: Yongli Peng, The Third Department of Orthopedics, People's Hospital of Renqiu, The Connor of Yu-Huan Road and XiHuan Road, Renqiu City, Hebei Province 062550, China, Email: pengyongli@163.com

\section{Abstract}

Objective: To review the progress of the application of dynamic assay of plasma D-dimer in orthopedic trauma patients for early diagnosis for concurrent deep vein thrombosis.

Methods: The literatures of venous thrombosis and D-dimer in the perioperative period of orthopedic trauma surgery were reviewed and analyzed.

Results: Dynamic monitoring of D-dimer of patients with lower extremity fractures during the perioperative period was of value for early diagnosis for concurrent deep vein thrombosis.

Conclusion: D-dimer assay in orthopedic trauma patients has important clinical value and significance in determining the occurrence of pulmonary embolism and reducing the perioperative mortality of patients.

Keywords: D-dimer; Venous thrombosis; Traumatology; Perioperative period

\section{Introduction}

The incidence of deep venous thrombosis (DVT) is high in orthopedic trauma patients and has become one of the common complications among orthopedic patients, the worst case of which is fatal pulmonary embolism that can cause unexpected peri operative death. Currently it is recognized that slow venous blood reflux, vascular wall injury and hyper coagulable blood state are the three major factors causing venous thrombosis [1]. Traumatic fracture can cause extensive damage to the vascular wall of the lower limb, subsequently the vascular endothelial injury activates the endogenous coagulation mechanism, so that the platelet aggregation increases, the coagulation factor is activated, the fibrinolytic enzyme activity decreases, and concurrently the external coagulation system is activated. These result in the high coagulation state of the blood and thus the increased risk of DVT. Improving the blood hypercoagulable state would play a significant role in prevention of DVT [2].

D-dimer is the smallest fragment of fibrin hydrolysis via the fibrinolytic enzyme after the cross-linking of fibrin monomers by activating factor XIII. D-dimer is a specific molecular marker of thrombin activation and fibrinolysis and could be used asan indirect indicator of in vivo hyper coagulable state and thrombosis [3]. D-dimer is significantly higher in acute DVT patients, which can be used as the indicator for screening DVT for the first choice. D-dimer in plasma is often used for DVT exclusion in clinics due to its high sensitivity and easy detection [4]. DVT formation is a dynamic process. The level of D-dimer in plasma after trauma is subject to dynamic changes. Dynamically monitoring the D-dimer levels in plasma in patients after traumatic surgery is therefore of value for early diagnosis for postoperative complication of DVT.

\section{Current research on D-dimer in orthopaedics}

Since its discovery, D-dimer has attracted a lot of interests in both the basic and clinical research. The research on D-dimer in orthopedics is highlighted in the following aspects:

Exclusion of population of potential thromboembolism: D-dimer levels in patients with DVT have been found significantly higher [5,6], interestingly the levels of D-dimer in plasma in patients of some other diseases also showed increase [7-9]. This indicates that D-dimer has a high sensitivity but low specificity for DVT diagnosis. Therefore, some researchers assessed the use of D-dimer as a negative predictive indicatorin DVT. Clinical studies $[10,11]$ reported that negative D-dimer in combination with low the pretest clinical probability (PTP)scores [12] could safely exclude $30 \%-50 \%$ of suspected DVT. In 2009, Yamaki et al. [13] performed PTP scoring and D-dimer assay for 989 patients with suspected thrombosis. These patients were divided into three groups of low, medium and high risks of thrombosis based on their PTP scores and the appropriate D-dimer cutoff value 
for each PTP group was set at the negative predictive value of $>98 \%$. The results showed that low PTP scores with appropriate D-dimer levels worked efficiently for excluding DVT. Moreover, this method was still safe for excluding DVT for the group of medium risk after raising the cut-off value of D-dimer.

Thrombosis recurrence prediction and risk assessment: By monitoring the D-dimer levels in patients of venous thrombosis, it was found that the levels of D-dimer in patients with recurrent thrombosis were statistically higher than those in patients without recurrent thrombosis [14].

Relationship with the severity of trauma: A retrospective study of 1049 patients, of which 548 were of acute trauma and 501 were of non-trauma, showed that the D-dimer levels of acute trauma patients were statistically significantly higher than those of the non-acute trauma patients and the D-dimer level showed positive correlation with the number of fractures [15].

Guidance for the timing for anticoagulants: Patients with venous thrombosis need to undergo prolonged anticoagulant therapy to prevent thrombosis recurrence and fatal pulmonary embolism. However, there is no guideline available to guide how long these patients need anticoagulant therapy. In 2014, Gualtiero Palareti et al. [16] reported the use of D-dimer assay to guide the timing for anticoagulant for venous thrombosis patients based on a 2-years follow-up treatment study of 1010 outpatients. Of these patients, 528 patients that showed continuously negative D-dimer was stopped anticoagulant therapy, as the result, 25 patients experienced thrombosis recurrence, the thrombosis recurrence rate was therefore $3.0 \%$. Of the remaining 482 patients, three hundred and seventy three continued to use anticoagulant, as the result, the thrombosis recurrence rate was $0.7 \%$; the rest 109 patients that refused to anticoagulant therapyhad $8.8 \%$ of thrombosis recurrence rate. These results indicated that continuously monitoring the D-dimer levels of venous thrombosis patients could be an effective approach to guiding the duration of the use of anticoagulant.

Guidance for the dosage of anticoagulants: The preventive medication of postoperative DVT is diverse, and there are no guidelines available for the use of the dosage regimens based on different diseases and populations. Dirk Peetzet al. [17] grouped the patients into low-risk and high-risk DVT according to their D-dimer levels, and the high-risk patients were given low molecular weight heparin (LMWH) twice a day, and lowrisk group once a day. The results showed that this strategy effectively prevented the occurrence of DVT.

\section{Future research on D-dimer in orthopaedic trauma}

Despite the above adverse effects, D-dimer is still the most potential laboratory marker for DVT in ortho paedic trauma. According to the latest American Academy of Orthopaedic Surgeons (AAOS) and American College of Chest Physicians (ACCP) guidelines, it is recommended that the patients with total knee and total hip arthroplasty or suspected DVT should first get the plasma D-dimer assayed, and the D-dimer assay in combination with the PTP score will then be used to determine whether or not colour Doppler and venous angiography are required. Further research may include the following areas:

A. Investigate the factors that affect D-dimer level, study how to effectively eliminate the effects of non-thrombotic factors, set up the personalized assay of D-dimer, and determine the personalized predictive diagnostic cut-off value of D-dimer and personalized clinical likelihood score program according to the specific circumstances of the patients to improve the role of D-dimer in clinical practice.

B. Analyse the trend of the dynamic change of D-dimer after orthopedic trauma surgery and detect the appropriate time point of of high thrombosis to improve the diagnostic accuracy.

C. Investigate additional markers to overcome the false positive issue of D-dimer due to its high sensitivity. The combined analysis of these markers would enhance the accuracy of DVT prediction.

\section{Conclusion}

In patients with orthopedic trauma surgery, venous thrombosis of the lower extremity is a common risk factor for pulmonary embolism. For patients with continuous increase in D-dimer level during perioperative surgery, further intravenous B-mode ultrasonography and angiography should be carried out. Patients with thrombosis and clinical manifestations should be promptly treated with physical, medication and implantation filter measures. Therefore, D-dimer detection of perioperative period in patients with lower extremity fractures has important clinical significance in diagnosingthe occurrence of pulmonary embolism and reducing the perioperative mortality of patients.

\section{References}

1. Anderson FA, Spencer FA (2010) Risk factors for venous thromboembolism. Clinics in Chest Medicine 107(1): 9-16.

2. Mont MA, Jacobs JJ (2012) AAOS clinical practice guideline: preventing venous thromboembolic disease in patients undergoing elective hip and knee arthroplasty. J Am Acad Orthop Surg 19(12): 777-778.

3. Yoo MC, Cho YJ, Ghanem E (2009) Deep vein thrombosis after total hip arthroplasty in Korean patients and D-dimer as a screening tool. Archives of Orthopaedic and Trauma Surgery 129(7): 887-894.

4. Becattini C, Lignani A, Masotti L (2012) D-dimer for risk stratification in patients with acute pulmonary embolism. Journal of Thrombosis and Thrombolysis 33(1): 48-57.

5. Yoshiiwa T, Miyazaki M, Takita C, Itonaga I, Tsumura H (2011) Analysis of measured D-dimer levels for detection of deep venous thrombosis and pulmonary embolism after spinal surgery. Journal of Spinal Disorders \& Techniques 24(4): E35-E39.

6. Ho CH (2011) Can very high level of D-dimer exclusively predict the presence of thromboembolic diseases? Journal of the Chinese Medical Association 74(4): 151-154. 
7. Montaner J, Mendioroz M, Ribó M, Delgado P, Quintana M, et al (2011) A panel of biomarkers including caspase-3 and D-dimer may differentiate acute stroke from stroke-mimicking conditions in the emergency department. Journal of Internal Medicine 270(2): 166-174.

8. Wakabayashi I, Masuda H (2009) Association of D-dimer with microalbuminuria in patients with type 2 diabetes mellitus. Journal of Thrombosis and Thrombolysis 27(1): 29-35.

9. Saray A, Mesihovic R, Gornjakovic S, Vanis N, Mehmedovic A, et al. (2012) Association between high D-dimer plasma levels and ascites in patients with liver cirrhosis. Medical Archives 66(6): 372-374

10. Kearon C, Ginsberg JS, Douketis J, Crowther M, Brill-Edwards P, et al. (2001) Management of Suspected Deep Venous Thrombosis in Outpatients by Using Clinical Assessment and d-dimer Testing. Annals of Internal Medicine 135(2): 108-111.

11. Anderson DR, Kovacs MJ, Kovacs G, Stiell I, Mitchell M, et al. (2003) Combined use of clinical assessment and d-dimer to improve the management of patients presenting to the emergency department with suspected deep vein thrombosis (the EDITED Study). J Thromb Haemost 1(4): 645-651.

12. Wells PS, Anderson DR, Bormanis J, Guy F, Mitchell M, et al. (2012) Value of assessment of pretest probability of deep-vein thrombosis in clinical management. Lancet 350(9094): 1795-1798.
13. Yamaki T, Nozaki M, Sakurai H (2009) Combined use of pretest clinical probability score and latex agglutination D-dimer testing for excluding acute deep vein thrombosis. Journal of Vascular Surgery 50(5): 10991105.

14. Poli D, Cenci C, Antonucci E, Grifoni E, Arcangeli C, et al. (2013) Risk of recurrence in patients with pulmonary embolism: predictive role of D-dimer and of residual perfusion defects on lung scintigraphy. Thromb Haemost 109(2): 181-186.

15. Zhang LD, Liu HB, Li YN, Ma HM, Liu YB, et al. (2012) Correlation analysis between plasma D-dimer levels and orthopedic trauma severity. Chin Med J (Engl) 125(17): 3133-3136.

16. Palareti G, Cosmi B, Legnani C, Antonucci E, De Micheli V, et al. (2014) D-dimer to guide the duration of anticoagulation in patients with venous thromboembolism: a management study. Blood 124(2): 196203.

17. Peetz D, Hafner G, Hansen M, Mayer A, Rippin G, et al. (2000) DoseAdjusted Thrombosis Prophylaxis in Trauma Surgery According to Levels of D-Dimer. Thrombosis Research 98(6): 473-483.

\section{Your next submission with Juniper Publishers will reach you the below assets}

- Quality Editorial service

- Swift Peer Review

- Reprints availability

- E-prints Service

- Manuscript Podcast for convenient understanding

- Global attainment for your research

- Manuscript accessibility in different formats

( Pdf, E-pub, Full Text, Audio)

- Unceasing customer service

Track the below URL for one-step submission https://juniperpublishers.com/online-submission.php 